\title{
ORIGINAL
}

\section{LA TIROTROPINEMIA (TSH) NEONATAL COMO INDICADOR DEL ESTADO NUTRICIONAL DE YODO EN CASTELLÓN Y VALENCIA (2004-2006)}

\author{
Carmen Barona-Vilar, Rosa Mas-Pons y Ana Fullana-Montoro
}

Servici de Salut Infantil i de la Dona. Direcció General de Salut Pública. Conselleria de Sanitat-Generalitat Valenciana.

\section{RESUMEN}

Fundamentos. En el cribado de hipotiroidismo congénito en poblaciones con ingesta suficiente de yodo la proporción de neonatos con valores de $\mathrm{TSH}>5 \mathrm{mU} / \mathrm{l}$ debe ser inferior al $3 \%$. El objetivo de este trabajo es conocer la prevalencia de déficit de yodo entre las madres y recién nacidos de Castellón y Valencia y, de manera secundaria, evaluar la influencia del día de obtención de la muestra y la utilización de antisépticos yodados.

Métodos. Se estudió el valor de TSH en 91.853 recién nacidos entre 2004 y 2006 en Castellón y Valencia. Se compararon las medianas de los valores de TSH considerando las condiciones que define la OMS para analizar el indicador: toma de muestra tras 2 días de vida, y no utilización de antisépticos yodados. Se calcularon y compararon las proporciones de muestras con TSH $>5 \mathrm{mU} / 1$.

Resultados. Entre las muestras que cumplieron las condiciones para el análisis del indicador, la prevalencia de neonatos con TSH $>5 \mathrm{mlU} /$ fue decreciente entre $2004 \quad(2,2 \%$ IC95\%:1,8\%-2,6\%), 2005 (2\%; IC95\%:1,6\%-2,3\%) y 2006 $(1,7 \%$; IC95\%: $1,4 \%-2 \%)$. La mediana de TSH en muestras de menos de 2 días fue significativamente superior $(2,19 \mathrm{mlU} / \mathrm{L}$; Q1-Q3: 1,35-3,40 frente a 1,36 mlU/L; Q1-Q3: 0,78-2,21) (p< 0,001 ). Considerando las muestras obtenidas tras 2 días de vida, el uso de antisépticos yodados determinó una mediana de TSH significativamente superior (1,54 miU/L; Q1-Q3: 0,882,50 frente a 1,23 mlU/L; Q1-Q3: 0,72-1,97) ( $<<0,001)$.

Conclusiones. El valor del indicador de tirotropinemia neonatal en Castellón y Valencia es compatible con la definición de la OMS para poblaciones con una ingesta adecuada de yodo. El cribado neonatal de TSH es una buena herramienta para monitorizar la prevalencia de déficit de yodo, pero debe adecuarse el momento de la extracción y eliminarse la utilización perinatal de antisépticos yodados.

Palabras clave: Hormonas tiroideas. Yodo. Hipotiroidismo congénito. Embarazo. Cribado neonatal.

Correspondencia:

Carmen Barona-Vilar

Unitat de Salut Perinatal

Direcció General de Salut Pública

Conselleria de Sanitat

C/ Micer Mascó, 31

46010 València

Correo electrónico: barona_car@gva.es

\section{ABSTRACT}

\section{Neonatal Thyrotropinemia (tsh) as an Indicator of Iodine Nutritional Level in Castellon and Valencia, Spain (2004-2006)}

Background: In the congenital hypothyroidism screening in populations with sufficient iodine intake, in the percentage of newborns with TSH values $5 \mathrm{mU} / \mathrm{l}$ must be less than $3 \%$. This study is aimed at ascertaining the prevalence of iodine deficiency among the mothers and newborns in Castellon and Valencia and, secondly, to evaluate the bearing which the day on which the sample is taken and the use of iodized antiseptics may have.

Methods: The TSH value was studied in 91,853 newborns within the 2004-2006 period in Castellon and Valencia. The mean TSH values were compared considering the conditions defined by the WHO for analyzing the indicator: sampling at two days following birth and no use of antiseptics. The percentages of samples having $\mathrm{TSH}>5 \mathrm{mU} / \mathrm{l}$.were calculated and compared.

Results: Among the samples having fulfilled the requirements for the analysis of the indicator, the prevalence of newborns having TSH $>5 \mathrm{mlU} /$ showed a decrease from 2004 (2.2\% CI95\%:1.8\%-2.6\%), 2005 (2\%; CI95\%:1.6\%-2.3\%) and 2006 (1.7\%; CI95\%: $1.4 \%-2 \%)$. The mean TSH in samples of under 2 days was significantly higher $(2.19 \mathrm{mlU} / \mathrm{L}$; Q1-Q3: $1.35-3.40$ as compared to $1.36 \mathrm{mlU} / \mathrm{L}$; Q1-Q3: 0.782.21) $(\mathrm{p}<0,001)$. Considering the samples taken 2 days after birth, the use of antiseptics determined a significantly higher mean TSH (1.54 mlU/L; Q1-Q3: $0.88-2.50$ as compared to $1.23 \mathrm{mlU} / \mathrm{L}$; Q1-Q3: 0.72-1.97) $(\mathrm{p}<0,001)$.

Conclusions: The value of the newborn thyrotropinemia indicator in Castellon and Valencia is compatible with the WHO definition for populations having a proper iodine intake. Neonatal TSH screening is a good tool for monitoring the prevalence of iodine deficiency, but the point in time of the extraction must be suitable and the use of iodized perinatal antiseptics eliminated.

Key words: Thyroid hormones. Iodine. Congenital hypothyroidism. Pregnancy. Neonatal screening. 


\section{INTRODUCCIÓN}

La deficiencia de yodo se considera un importante problema de salud pública, especialmente en el grupo de población de mujeres embarazadas y en los niños. Un aporte adecuado de yodo durante la gestación e incluso en la época previa a la concepción es fundamental para el desarrollo cerebral e intelectual del niño ${ }^{1-4}$. Por ello, la monitorización del estado nutricional de yodo en una determinada comunidad resulta esencial para poder llevar a cabo medidas colectivas de carácter preventivo ${ }^{5}$.

Para conocer la situación epidemiológica del estado nutricional de yodo en una determinada comunidad, la OMS y el ICCIDD 5 han propuesto como indicadores la prevalencia de bocio y la yoduria en la población escolar, la tiroglobulina y la prevalencia de hipertirotropinemia neonatal (TSH $>5$ $\mathrm{mU} / \mathrm{l})$ en las pruebas de cribado del hipotiroidismo congénito. La prevalencia de bocio es un buen indicador para analizar la situación de partida, pero presenta limitaciones para medir el impacto de las intervenciones, ya que es probable que el tamaño del tiroides no vuelva a la normalidad hasta pasados meses e incluso años de la corrección del déficit. Por ello, en estos momentos se recomienda realizar estudios poblacionales de yodurias cada 3-5 años, en los que se incluyan diferentes grupos de población, pues los niveles de yodo en la orina permiten evaluar la ingesta reciente de yodo ${ }^{6}$.

España ha sido considerada tradicionalmente como una zona con deficiencia leve o moderada de yodo $^{7,8}$, apoyándose esta consideración mayoritariamente en el análisis de la prevalencia de bocio y la yoduria en escolares. También se han llevado a cabo estudios en algunos lugares de España basados en la yoduria materna, que parecen indicar una ingesta insuficiente de yodo durante la gestación ${ }^{9-12}$. Sin embargo, el último informe de la OMS, a partir de la revisión sistemática de las publicaciones llevadas a cabo durante el periodo 19932003, considera que España está entre los países que presentan un estado nutricional adecuado de yodo ${ }^{13}$. Este cambio significativo en el grado de yodación de la población, medido a través del indicador de yodurias en escolares, también lo corroboran algunos trabajos recientes llevados a cabo en Asturias ${ }^{14}$, Cataluña ${ }^{15,16}$, Andalucía $^{17,18}$ y Comunitat Valenciana ${ }^{19,20}$.

El valor de la TSH neonatal es un indicador particularmente sensible del estado nutricional de yodo durante el embarazo y en los recién nacidos, y se considera una herramienta muy útil para monitorizar la evolución de la situación en los países con deficiencia leve o moderada de yodo $^{21,22}$. Sin embargo, en España son escasos los estudios centrados en analizar el valor de la TSH neonatal para valorar el estado nutricional de yodo en el embarazo y recién nacidos. Para poder utilizar este indicador es necesario tener implantado un sistema de cribado neonatal de hipotiroidismo congénito, con garantías de que la muestra se haya tomado pasadas 48 horas de vida para evitar el incremento de TSH fisiológico que se produce en las primeras horas de vida $^{22,23}$. Para que el indicador sea válido también debe de tenerse en cuenta que no se hayan utilizado antisépticos yodados en el período perinatal ${ }^{5,22,24}$. En poblaciones con una ingesta de yodo suficiente la proporción de neonatos con valores de $\mathrm{TSH}>5$ $\mathrm{mU} / \mathrm{l}$ debe ser inferior al 3\%. Una frecuencia del 3\%-19\% indica deficiencia leve, entre el 20\%-39,9\% moderada, y por encima del $40 \%$ severa ${ }^{5}$.

El objetivo de este estudio es conocer la prevalencia de déficit de yodo entre las mujeres y sus hijos recién nacidos de Castellón y Valencia, a partir del programa de cribado neonatal de hipotiroidismo congénito y valorar cuáles son las condiciones adecuadas para su utilización como sistema de monitorización en nuestro entorno. 


\section{SUJETOS Y MÉTODOS}

Estudio transversal realizado a partir de los datos obtenidos en el Programa de Cribado de Enfermedades Metabólicas Congénitas, en el que se recoge el valor de la TSH determinada en una muestra de sangre extraída del talón de los recién nacidos. Este registro también incluye variables de carácter sociodemográfico (edad y país de origen de la madre, sexo del bebé y fecha de nacimiento), obstétricas (semanas de gestación, tipo de parto, peso del recién nacido, gemelaridad y tipo de lactancia), así como datos de identificación del hospital donde se extrae la muestra y momento de su realización (días transcurridos entre el nacimiento y la extracción).

La población de estudio la integraron 91.853 recién nacidos en las maternidades públicas y privadas de Castellón y Valencia, de los cuales 25.078 nacieron en 2004, 32.607 en 2005 y 34.168 en 2006. La estrategia de cribado en la Comunitat Valenciana desde el año 2000 consiste en la extracción de una doble muestra. La primera, para la detección de TSH, se toma antes del alta de la maternidad, preferentemente pasadas 48 horas de vida, con la finalidad de conseguir una cobertura óptima. La segunda, para la detección de fenilalanina, se realiza en los centros de salud, preferentemente al $5^{\circ}$ $7^{\circ}$ día de vida. Las muestras se envían siempre a través de mensajería al laboratorio de referencia ${ }^{25}$.

La determinación de TSH en sangre entera seca en papel de filtro se realizó en el Laboratorio de Metabolopatías del Hospital Universitario La Fe de Valencia por el método de inmunofluorescencia a tiempo retardado (AutoDELFIA, Perkin Elmer/Wallac, Turku, Finlandia). Los límites de detección de la técnica se sitúan entre 0,2 y $250 \mathrm{mU} / \mathrm{mL}$, en los estándares usados el coeficiente de variación intraensayo fue 6.1, 4.2, 5.2 e interensayo 9, 9.5, 6.7, la sensibilidad del $100 \%$ y la especificidad del
99\%. No se utilizaron muestras extraídas de sangre de cordón. En 2001 la Dirección General de Salud Pública puso en marcha una aplicación informática que transcribe los valores de TSH automáticamente desde la máquina analizadora (Mult Calc) a la base de datos informatizada, en la que se suman al resto de variables de identificación del recién nacido.

Se identificaron los casos en los que la muestra se tomó antes y después de transcurridos dos días desde el nacimiento, para determinar el efecto del momento de la toma de muestra sobre los valores de TSH. Del mismo modo se realizó una encuesta telefónica a todas las maternidades públicas y privadas para identificar aquéllas en las que en el período de estudio se hubiera aplicado algún tipo de antiséptico yodado, en la madre o en el recién nacido, con la finalidad de determinar el efecto de esta práctica sobre los valores de la TSH neonatal. Se excluyeron del análisis 14 casos de hipotiroidismo congénito diagnosticados a partir del cribado en 2004, 18 en 2005 y 21 en 2006. Tal como recomienda la OMS, se consideró una prevalencia superior al $3 \%$ del valor de $\mathrm{TSH}>5 \mathrm{mU} / \mathrm{l}$ para definir la situación de deficiencia leve-moderada de yodo ${ }^{5}$.

Para el análisis estadístico se obtuvieron las medianas de los valores de TSH con sus rangos intercuartiles. El análisis de las diferencias entre medianas se llevó a cabo utilizando los tests no paramétricos de U Mann_Whitney y Kruskal-Wallis y se recurrió al test de chi $^{2}$ para estudiar las diferencias entre proporciones. El análisis estadístico se llevó a cabo utilizando los programas SPSS/PC+ (versión 14.0) y EPIINFO (versión 3).

\section{RESULTADOS}

La cobertura del programa de cribado fue superior al $98 \%$ en cada uno de los años 
del estudio. De los 91.853 neonatos a los que se realizó el cribado de hipotiroidismo congénito entre 2004 y 2006 el 51\% eran niños y el $49 \%$ niñas. El 90,7\% había nacido a término, frente a un $9,3 \%$ con menos de 37 semanas de gestación en el momento del parto. Contaban con pesos de 2.500 grs. o superiores el $91 \%$ y un 3,8\% eran gemelos. Las madres tenían una edad media de 31 años (DE 5), el 75\% dieron a luz mediante un parto vaginal y el $80,5 \%$ optó por la lactancia materna.

Respecto al momento de realización del cribado (tabla 1) en el 57,3\% de los casos la muestra se obtuvo antes de los 2 primeros días de vida frente al $42,7 \%$ que fue tras este período. A través de la encuesta realizada a las 17 maternidades (13 públicas y 4 privadas) en las que se tomaron las muestras, se constató que 11 en 2004, 10 en 2005 y 8 en 2006 habían utilizado antisépticos yodados en algún momento sobre la piel o mucosas de la madre (anestesia epidural, episiotomía, herida de cesárea, lavados vaginales), siendo generalizada la utilización de alcohol sobre el cordón umbilical. Las condiciones óptimas (prueba tras dos días de vida y no uso de antisépticos yodados) para poder analizar el indicador de hipertirotropinemia neonatal se cumplieron en el $22,6 \%$ de la muestra (20.747 neonatos) tal como refleja la tabla 1 .

En los neonatos a los que se les practicó la toma de muestra en los primeros 2 días de vida, los valores de la mediana de TSH fueron superiores respecto a aquéllos en los que la prueba de cribado se obtuvo pasados 2 días de vida. (2,19 mlU/L; Q1-Q3: 1,353,40 $\mathrm{mlU} / \mathrm{L}$ frente a 1,36 $\mathrm{mlU} / \mathrm{L} ; \mathrm{Q} 1-\mathrm{Q} 3$ : $0,78-2,21 \mathrm{mlU} / \mathrm{L})$. La prevalencia de neonatos con valores de $\mathrm{TSH}>5 \mathrm{mU} / \mathrm{l}$ en el primer grupo resultó significativamente superior al alcanzar al 9,3\% (IC95\%: 9,0-9,6\%) frente al 2,9\% ( IC95\%: 2,7-3,1\%) de los casos en que la muestra se obtuvo pasados dos días (tabla 2).

Tabla 1

Distribución de la muestra según las condiciones en las que se realizó el cribado

\begin{tabular}{|c|c|c|c|c|c|c|c|c|c|c|}
\hline \multirow{3}{*}{ Año } & \multicolumn{4}{|c|}{ Días nacimiento-extracción } & \multicolumn{4}{|c|}{ Antisépticos yodados } & \multirow{2}{*}{\multicolumn{2}{|c|}{$\mathbf{N}^{\circ} \underset{\text { muestras váli- }}{\text { das }}$}} \\
\hline & \multicolumn{2}{|c|}{$<=2$} & \multicolumn{2}{|c|}{$>2$} & \multicolumn{2}{|c|}{ Sí } & \multicolumn{2}{|c|}{ No } & & \\
\hline & $\mathbf{N}$ & $\%$ & $\mathbf{N}$ & $\%$ & $\mathbf{N}$ & $\%$ & $\mathbf{N}$ & $\%$ & $\mathbf{N}$ & $\%$ \\
\hline 2004 & 13.407 & 54,7 & 11.115 & 45,3 & 16.385 & 65,3 & 8.693 & 34,7 & 5.312 & 21,2 \\
\hline 2005 & 18.910 & 58,2 & 13.561 & 41,8 & 18.861 & 57,8 & 13.746 & 42,2 & 6.697 & 20,5 \\
\hline 2006 & 19.611 & 58,2 & 14.064 & 41,8 & 16.419 & 48,1 & 17.749 & 51,9 & 8.738 & 25,6 \\
\hline Total & 51.928 & 57,3 & 38.740 & 42,7 & 51.665 & 56,2 & 40.188 & 43,8 & 20.747 & 22,6 \\
\hline
\end{tabular}

Tabla 2

Distribución de la concentración de TSH (mIU/L) neonatal según el momento de extracción de la muestra y uso de antisépticos yodados

\begin{tabular}{|c|c|c|c|c|}
\hline & $\begin{array}{c}\text { Mediana } \\
\text { (Rango Intercuartil) }\end{array}$ & $\mathbf{P}^{*}$ & $\begin{array}{c}\% \text { Neonatos con valor } \\
\text { de } \mathrm{TSH}>5 \mathrm{mIU} / \mathrm{L} \text { (IC } 95 \% \text { ) }\end{array}$ & $\mathbf{P} * *$ \\
\hline $\begin{array}{l}<=\mathbf{2} \text { días de vida } \\
(\mathrm{N}=51.928)\end{array}$ & $\begin{array}{c}2,19 \\
(1,35-3,40)\end{array}$ & \multirow{2}{*}{$<0,001$} & $\begin{array}{c}9,3 \\
(9,0-9,6)\end{array}$ & \multirow{2}{*}{$<0,001$} \\
\hline $\begin{array}{l}>2 \text { días de vida } \\
(\mathrm{N}=38.740)\end{array}$ & $\begin{array}{c}1,36 \\
(0,78-2,21)\end{array}$ & & $\begin{array}{c}2,9 \\
(2,7-3,1)\end{array}$ & \\
\hline $\begin{array}{l}\text { > } 2 \text { días de vida/ } \\
\text { Sí } \mathbf{A Y}(\mathrm{N}=17.446)\end{array}$ & $\begin{array}{c}1,54 \\
(0,88-2,50)\end{array}$ & \multirow{2}{*}{$<0,001$} & $\begin{array}{c}4,0 \\
(3,7-4,3)\end{array}$ & \multirow{2}{*}{$<0,001$} \\
\hline $\begin{array}{l}>2 \text { días de vida/ } \\
\text { No } \mathrm{AY}(\mathrm{N}=20.747)\end{array}$ & $\begin{array}{c}1,23 \\
(0,72-1,97)\end{array}$ & & $\begin{array}{c}1,9 \\
(1,7-2,1)\end{array}$ & \\
\hline $\begin{array}{l}\text { P*: U Mann_Whitney } \\
\mathrm{P}^{* *}: \mathrm{chi}^{2}\end{array}$ & & & & \\
\hline
\end{tabular}


Tras controlar el efecto de la toma de muestra en los dos primeros días de vida, el uso de antisépticos yodados implicó valores de la mediana de TSH superiores $(1,54$ $\mathrm{mU} / \mathrm{L}$ Q1-Q3:0,88-2,5 mlU/L frente a 1,23 mlU/L Q1-Q3:0,72-1,97 mlU/L; P<0,001). Así, en el grupo de nacidos en maternidades donde se usaron antisépticos yodados la prevalencia de neonatos con $\mathrm{TSH}>5 \mathrm{mU} / \mathrm{l}$ fue significativamente mayor $(4 \%$ IC95\%: $3,7-4,3 \%$ frente al $1,9 \%$ IC $95 \%: 1,7-2,1 \%$; $\mathrm{P}<0,001$ ) (tabla 2).

En condiciones óptimas para analizar el indicador de hipertirotropinemia neonatal (maternidades que no utilizaron antisépticos yodados y que realizaron la prueba de cribado pasadas 48 horas de vida), la prevalencia global de neonatos con TSH $>5$ mlU/L fue del 1,9\%, (IC95\%:1,7\%-2,1\%), observando además una tendencia descendente, aunque no significativa entre 2004 $(2,2 \%$ IC95\%:1,8\%-2,6\%), 2005 (2\%; IC95\%:1,6\%-2,3\%) у $2006(1,7 \%$; IC95\%: 1,4\%-2\%). Sin embargo la evolución decreciente de los valores de la mediana de TSH en los tres años, sí que presentó diferencias significativas $(\mathrm{p}<0,001)$ (figura 1$)$.

Las maternidades que cumplieron los criterios para el análisis (figura 2) muestran una disminución de la prevalencia de neonatos con TSH>5 mlU/L entre 2004 y 2006 en todas las maternidades, salvo en la M3 y

Figura 1

Evolución anual del valor de la mediana de TSH (mU/L)

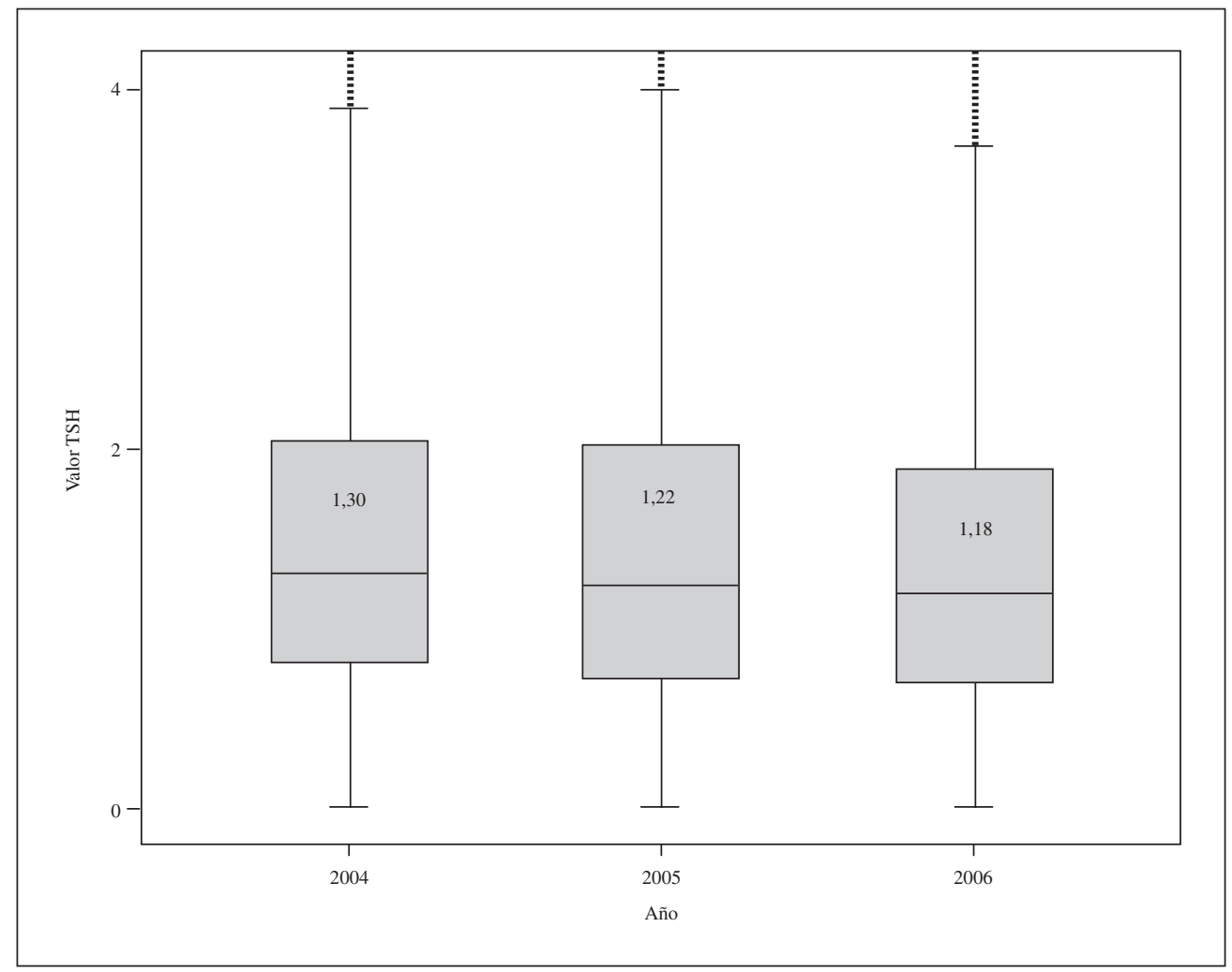

$\mathrm{p}<0,001$. Kruskal-Wallis. 
Figura 2

Prevalencia de la concentración de $\mathrm{TSH}>5 \mathrm{mlU} / \mathrm{l}$ anual en las maternidades que cumplieron los requisitos para analizar el indicador

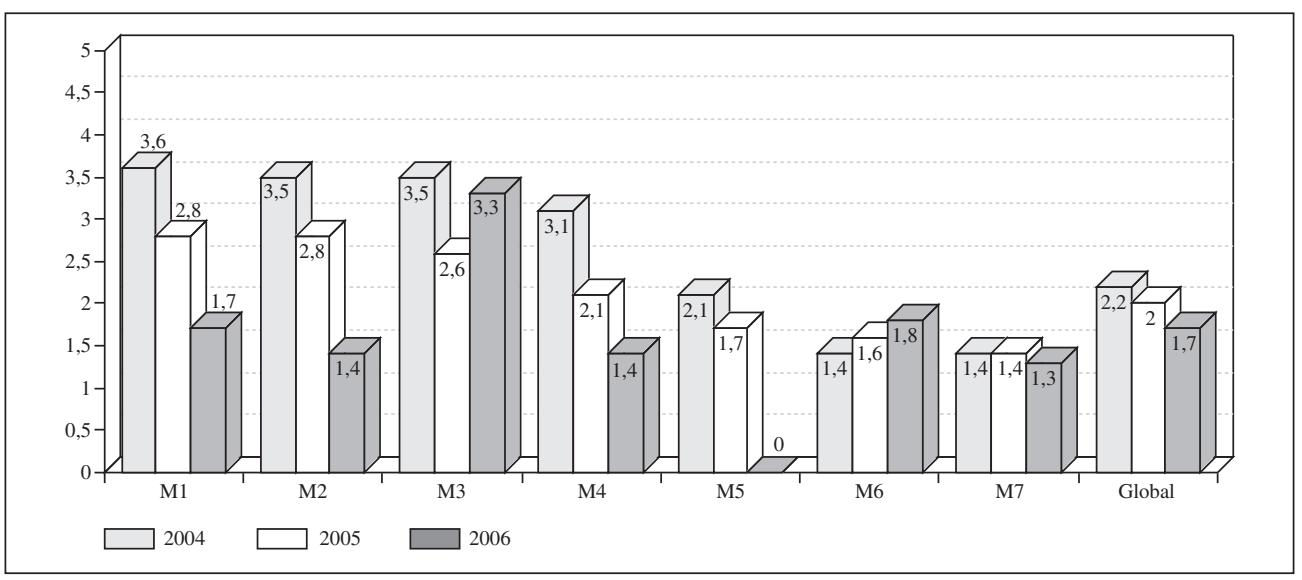

Figura 3

Comparación de los valores globales (2004-2006) de las medianas de TSH (mlU/l) en las diferentes maternidades que cumplieron los requisitos para analizar el indicador

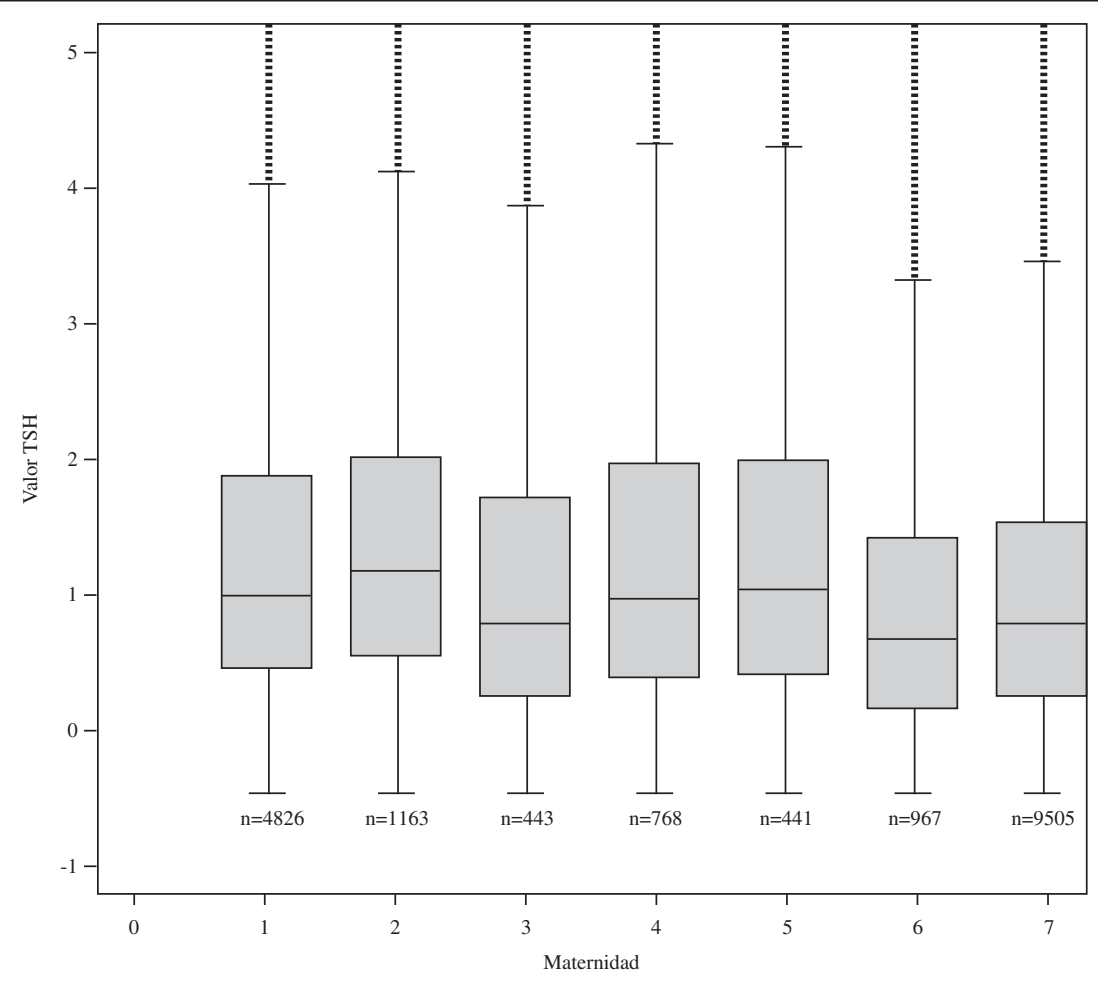

$\mathrm{p}<0,001$. Kruskal-Wallis. 
en la M6, aunque las diferencias interanuales no son estadísticamente significativas en ninguna maternidad. Sin embargo sí que resulta significativa la comparación de la mediana global de todo el período estudiado, entre las diferentes maternidades situadas en zonas geográficas diferentes de la comunidad $(\mathrm{p}<0,001)$ (figura 3$)$.

\section{DISCUSIÓN}

Los resultados de este estudio, llevado a cabo a partir del programa de cribado de hipotiroidismo congénito en los neonatos de las provincias de Castellón y Valencia, muestra una prevalencia de hipertirotropinemia neonatal compatible con la definición de la OMS para poblaciones con una ingesta adecuada de yodo. Además es compatible con los resultados obtenidos a partir del indicador de yodurias en escolares de dos estudios recientes llevados a cabo en la Comunitat Valenciana. El primero, en un área sanitaria que abarca cuatro comarcas del interior de la provincia de Valencia, obtuvo yodurias indicativas de una adecuada nutrición de yodo (155 $\mu \mathrm{g} / \mathrm{l}$ de mediana), aunque acompañada de una prevalencia de bocio del $33 \%{ }^{19}$. El segundo estudio, en una muestra representativa de los escolares de 6-11 años de la provincia de Alicante, también halló niveles de yodurias (188 $\mu \mathrm{g} / \mathrm{l}$ de mediana) que apuntan a una ingesta poblacional adecuada de yodo ${ }^{20}$.

La prevalencia de hipertirotropinemia neonatal obtenida en este estudio es inferior a la alcanzada en estudios similares llevados a cabo en Canarias $^{26}(9 \%)$ y Málaga ${ }^{17}$ (6,6\%-9,8\%). Desconocemos si la selección de la muestra en ambos casos fue rigurosa en relación con el uso de antisépticos yodados y si la de Málaga además lo fue con los neonatos de más de 2 días de vida. El presente estudio corrobora que si no se cumplen ambas condiciones el indicador de TSH neonatal queda claramente distorsionado al alza ${ }^{5}$.
Durante la gestación se produce un incremento de las necesidades de yodo ${ }^{27}$ que hace aconsejable dirigir la estrategia a conseguir una ingesta adecuada de yodo en toda la población en las regiones que presentan una deficiencia leve o moderada, para garantizar que las mujeres en edad fértil lleguen al embarazo en condiciones de nutrición adecuada de yodo ${ }^{28,29}$. Recomendaciones recientes de la OMS apuntan a que cuando el consumo de sal yodada se extiende a más del $90 \%$ de la población general durante al menos dos años, es razonable pensar que las necesidades de yodo de la población más vulnerable, como son las mujeres en edad fértil y las mujeres embarazadas y las lactantes, estarán cubiertas por la $\operatorname{dieta}^{30}$. Otras medidas como la suplementación farmacológica podrían beneficiar a algunas mujeres, pero sería innecesaria en las que lleguen al embarazo en condiciones de yodosuficiencia ${ }^{6,27}$.

Una limitación derivada de las características de la fuente utilizada en este estudio es que los valores de TSH neonatal hallados no se pueden contrastar con información sobre la ingesta materna de yodo en forma de alimentos o suplementos. La recomendación a todas las mujeres embarazadas de consumir sal yodada se recogió en el Programa de Control Básico del Embarazo en $2001^{31}$ y los estudios previos en escolares de nuestra comunidad sitúan este consumo en torno al $70 \%$ de los hogares ${ }^{19,20}$. Respecto a la suplementación farmacológica, el único preparado del mercado utilizado como suplemento de yodo comenzó su venta en 2005, segundo año de nuestro estudio, con una baja dispensación (2.477 envases), si se tiene en cuenta que ese año hubo 50.954 nacimientos. La cifra de ventas en 2006 creció notablemente hasta 28.204, año en el que nacieron 52.803 niños/as, según datos de la Dirección General de Farmacia.

Las diferencias significativas en el indicador de hipertirotropinemia neonatal halladas entre maternidades que dan cober- 
tura a poblaciones de diferentes ámbitos geográficos de la Comunitat Valenciana, podrían obedecer a diferentes patrones regionales de consumo de las distintas fuentes de yodo (agua, leche, pescado, sal, suplementos farmacológicos). En este sentido harían falta estudios complementarios para poder contrastar las cifras de TSH neonatal halladas, con los niveles de ingesta de yodo maternos medidos a través de las yodurias y hormonas tiroideas maternas (TSH y T4L).

Dado que hasta la fecha no existen estudios representativos de todo el Estado Español, la implantación del cribado neonatal de hipotiroidismo congénito consolidada en todas las Comunidades Autónomas puede convertirlo en una herramienta eficaz y complementaria de los estudios sobre yodurias en escolares y otros grupos sensibles de población para la vigilancia epidemiológica del estado nutricional de yodo, permitiendo identificar posibles diferencias a nivel territorial. Para ello es necesario cumplir de manera rigurosa las condiciones para que el análisis sea válido, y en este estudio se pone de manifiesto el hecho apuntado en otras ocasiones sobre la variación en las cifras de TSH neonatal en función del momento de extracción de la muestra ${ }^{21,23}$ y por la influencia del uso de antisépticos yodados en la etapa perinatal ${ }^{5,24}$. A pesar de las recomendaciones, en este estudio todavía hubo un considerable porcentaje de neonatos a los que no se les realizó el cribado en condiciones óptimas, generando una situación de hipertirotropinemia transitoria (falsos positivos en la prueba de cribado), repetición de pruebas y la imposibilidad de utilizar esas muestras para monitorizar el estado nutricional de yodo.

Como conclusión, los resultados de este estudio sugieren que la Comunidad Valenciana no presenta déficit de yodo a nivel poblacional, de acuerdo con los criterios de clasificación de los valores de tirotropinemia neonatal establecidos por la OMS. El cribado neonatal de TSH es una buena herramienta para monitorizar el estado nutricional de yodo a nivel poblacional, pero es necesario adecuar el momento de la extracción, transcurridos dos días de vida, y eliminar la utilización perinatal de antisépticos yodados.

\section{BIBLIOGRAFÍA}

1. Delange F. Epidemiology and Impact of Iodine Deficiency in Pediatrics. J Pediatr Endocrinol Metab. 2005;18:1245-51.

2. Morreale de Escobar G, Obregón MJ and Escobar del Rey F. Is Neuropsychological Development Related to Maternal Hypothyroidism or Maternal Hypothyroxinemia?. J Clin Endocrinol Metab. 2000; 85:3975-87.

3. Glinoer D. Pregnancy and Iodine. Thyroid. 2001;11:471-81.

4. Haddow JE, Palomaki GE, Allan WC, Williams JR, Knight GJ,Gagnon J, et al. Maternal thyroid deficiency during pregnancy and subsequent neuropsychological development of the child. N Engl J Med. 1999;341:549-55.

5. WHO, UNICEF, ICCIDD. Assessment of the Iodine Deficiency Disorders and Monitoring their Elimination. Geneva: WHO/NHD/01.1; 2001.

6. ICCIDD. Iodine requirements in pregnancy and infancy. IDD Newsletter. 2007;23(1).

7. Vila L. La deficiencia de yodo en España: un problema pendiente que urge resolver. Endocrinol Nutr. 2002;49:1-4.

8. Díaz-Cardóniga FJ y Delgado-Álvarez (coordinadores). Déficit de yodo en España: situación actual. Endocrinol Nutr. 2004;51:2-13.

9. Almodóvar Ruiz F, Gorgojo Martínez JJ, Lahera Vargas M, Cava Valenciano f, Valor García S, Donnay Candil S. Déficit de yodo en una población de mujeres embarazadas pertenecientes a un área con leve deficiencia de yodo. Endocrinol Nutr. 2006;53:577-81.

10. Rodríguez I, luna R, Ríos M, Fluiters E, Páramo C y García-Mayor RV. Déficit de yodo en gestantes y mujeres en edad fértil pertenecientes a un área con consumo normal de yodo. Med Clin (Barc). 2002;118:217-8. 
11. Vila L, Muñoz J, Casmitjana R, García A, Legaz G, Barrionuevo C, et al. y grupo GEDIG. Estudio de la deficiencia de yodo de la población gestante de los Pirineos. Endocrinol Nutr. 2002;49(supl 1):5.

12. González Mateo MC, Fernández Fernández M, Díez Hernández A, Delgado Gómez M, García Menéndez L y Díaz Cardóniga F. Bocio, función tiroidea y excreción de yodo en gestantes de la zona de El Bierzo. Endocrinol Nutr. 2002;49:28992.

13. WHO. Iodine Deficiency in Europe: A continuing public health problem. Geneva: WHO/UNICEF; 2007.

14. Delgado E, Díaz-Cardóniga FJ, Tartón T, Bobis ML, Valdés MM y Méndez A. Erradicación de los transtornos por deficiencia de yodo en Asturias (España): 18 años de yodoprofilaxis con sal. Endocrinol Nutr. 2004;51:492-6.

15. Serra-Prat M, Díaz E, Verde Y, Gost J, Serra E y Puig Domingo M. Prevalencia de déficit de yodo y factores asociados en escolares de 4 años. Med Clin (Barc). 2003;120:246-9.

16. Vila Ll, Castell C, Wengrowicz S, de Lara N y Casamitjana R. Estudio de la yoduria en la población adulta de Cataluña. Med Clin (Barc). 2006;127:730-3.

17. Gómez Huelga R, Millón MC, Soriguer F, Mancha I, Garriga MJ, Muñoz R, et al. Comparación de diferentes criterios de diagnóstico poblacional de la deficiencia de yodo (DDY). Prevalencia de bocio endémico en la Axarquía (Málaga). Endocrinol Nutr. 2000;47:260-6.

18. Madueño Caro AJ, Cabezas Saura PB, Díaz Orta J, Benítez Rodríguez E, Ruíz Galdón M y Gómez A. Prevalencia de bocio y deficiencia de yodo en población escolar de una zona básica de salud tradicionalmente endémica. Aten Primaria. 2001;27:258-62.

19. Peris Roig B, Atienzar Herráez N, Merchante Alfaro AA, Calvo Rigual F, Tenías Burillo JM, Selfa Moreno S, et al. Bocio endémico y déficit de yodo: ¿sigue siendo una realidad en España? An Pediatr (Barc). 2006;65:234-40.

20. Zubiaur Cantalapiedra A, Zapico Álvarez-Cascos MD, Ruiz Pérez L, Sanguino López L, Sánchez Serrano FJ, Alfayate Guerra R, et al. Situación nutricional de yodo en la población escolar de Alicante. An Pediatr (Barc). 2007;66:260-6.
21. Delange F. Screening for congenital hypothyroidism used as an indicator of the degree of iodine deficiency and of its control. Thyroid. 1998; $8: 1185-92$

22. Zimmermann MB, Aeberli I, Torresani T y Bürgi $\mathrm{H}$. Increasing the iodine concentration in the Swiss iodised salt program markedly improved iodine status in pregnant women and children: a 5y prospective national study. Am J Clin Nutr. 2005;82:388-92.

23. McElduff A, McElduff P, Gunton J, Hams G, Wiley $\mathrm{V}$ and Wilcken BM. Neonatal thyroid-stimulating hormone concentrations in northern Sydney: further indications of mild iodine deficiency?. Med J Aust. 2002;176:317-20.

24. Arena Ansotegui J y Emparanza Knörr LI. Los antisépticos yodados no son inocuos. An Esp Pediatr. 2000;53:25-9.

25. Generalitat Valenciana. Manual del Programa de Prevención de Minusvalías Psíquicas: Detección Precoz de Alteraciones Metabólicas Congénitas. Programa de metabolopatías. Valencia: Generalitat Valenciana; 2001.

26. Domenech Martínez E y Barroso Guerrero F. Utilidad del programa de cribado neonatal de hipotiroidismo congénito para la valoración de la deficiencia de yodo en Canarias. An Pediatr .2003;58:357-63.

27. Glinoer D. Thyroid Regulation and Dysfunction in the Pregnant Patient. Last revised: April 4, 2006. Disponible en: www.thyroidmanager.org/chapter14/index.html.

28. Pearce EN, Hollowell JG. Iodine Nutrition. More is Better. N Engl J Med 2006;355:1500.

29. Laurberg P, Nohr SB. Iodine intake and prevention of thyroid disorders: surveillance is needed. Med J Aust. 2002; 176:306-7.

30. WHO. Reaching optimal iodine nutrition in pregnant and lactating women and young children: programmatic recommendations (ed). Public Health Nutr. 2007;10:1527-29. doi: $10.1017 / \mathrm{S} 1368980007705360$.

31. Generalitat Valenciana. Control Básico del Embarazo en la Comunidad Valenciana. Manual para Profesionales Sanitarios. Valencia: Generalitat Valenciana. Conselleria de Sanitat; 2002. Sèrie E: Programes Sanitaris num 45. 
